\title{
Determinantes y consecuencias de la efectividad de las promociones basadas en precios
}

\section{Antecedents and consequences of price promotions effectiveness}

\author{
ISABel María Rosa Díaz ${ }^{1}$ \\ Francisco Javier Rondán CATALuña ${ }^{2}$ \\ Universidad de Sevilla (España)
}

Recibido el 30 de enero de 2010, aceptado el 13 de octubre de 2011

$\mathrm{N}^{\circ}$ de clasificación JEL: M31

DOI: $10.5295 /$ cdg.100207ir

\section{Resumen:}

Partiendo de un enfoque de orientación al mercado, el presente trabajo analiza la influencia que ejercen diversos factores sobre la efectividad de las promociones basadas en precios. Dichos factores están relacionados tanto con el comportamiento de compra (importancia del precio dentro del proceso de decisión y nivel de conocimiento de los precios que poseen los compradores) como con las características socioculturales y demográficas de los consumidores (sexo, nivel de formación y nivel de ingresos). El trabajo empírico desarrollado, basado en una muestra de 600 consumidores, ha permitido trazar un perfil que define a los consumidores más proclives a sentirse atraídos por los precios promocionales.

Palabras clave:

Promociones, precios, consumidor, comportamiento de compra.

\begin{abstract}
:
From an approach of market orientation, the present research analyzes the influence of several factors on the efficiency of price promotions. The above mentioned factors are related to purchase behavior (the importance of price in the decision process and the consumers' level of price knowledge), as well as to the sociocultural and demographic characteristics (gender, education level, and income level). The empirical study developed, based on a sample of 600 consumers, has allowed to draw a common profile for consumers who are attracted to price promotions sales.
\end{abstract}

Keywords:

Promotions, prices, consumer, purchase behavior.

\footnotetext{
${ }^{1}$ Departamento de Administración de Empresas y Marketing. Facultad de Ciencias Económicas y Empresariales. Avda. Ramón y Cajal, 1 - 41018 Sevilla.imrosa@us.es

${ }^{2}$ Departamento de Administración de Empresas y Marketing. Facultad de Ciencias Económicas y Empresariales Avda. Ramón y Cajal, 1 - 41018 Sevilla.rondan@us.es
} 


\section{INTRODUCCIÓN}

Las promociones basadas en precios son prácticas muy habituales, con las que las empresas persiguen diversos objetivos: dar salida a sus inventarios, atraer a nuevos consumidores que aún no conocen el producto, influir sobre su imagen de precios, y diferenciarse de sus competidores (Klapper et al., 2005; Palazón y Delgado, 2009; Shankar y Krishnamurthi, 1996). Pero, ¿resultan estas prácticas promocionales siempre eficaces y convenientes? ¿Atraen al público adecuado? ¿Qué elementos deben tenerse en cuenta para diseñar una promoción basada en precios?

La respuesta a los interrogantes anteriores no resulta sencilla, dado el gran número de factores implicados (Krider y Han, 2004; Palazón y Delgado, 2008; Rosa, 2004): a) la intensidad de la competencia en el mercado; b) las prácticas de precios más frecuentes; c) las categorías de productos y servicios considerados; d) las características socio-demográficas de los consumidores; e) las variables que definen los comportamientos de dichos consumidores.

Desde una perspectiva de orientación al mercado, los consumidores se distinguen entre sí en función de su personalidad, valores y preferencias (Martínez y Montaner, 2006), lo que les lleva a desarrollar comportamientos y reacciones diferentes ante los precios y sus modificaciones (Gázquez y Sánchez, 2009; Grewal et al., 2004; Vanhuele y Drèze, 2002). En consecuencia, las reacciones ante un estímulo -precio promocional- pueden variar según el nivel de importancia que atribuya a la decisión de compra -nivel de implicación del consumidor- (Campo y Yagüe, 2007; Estelami y Lehmann, 2001; Gázquez y Sánchez, 2009) y de las premisas de evaluación que utilice -percepciones, información y conocimiento disponible-(Binkley and Bejnarowicz, 2003; Campo y Yagüe, 2007; KukarKinney et al., 2007). Además, las reglas de decisión de los consumidores y su esfuerzo cognitivo y de búsqueda de información pueden modificarse en función del contexto de compra y de sus características personales (Ganesh et al., 2000; Homburg and Stock, 2005; Kukar-Kinney et al., 2007; Parreño et al., 2009).

El interés de esta cuestión se ve aumentado por un factor fundamental dentro de los estudios sobre comportamientos. Se trata del factor socio-cultural, pues la mayoría de los estudios sobre el comportamiento del consumidor ante los precios se han desarrollado en contextos sociales y culturales muy localizados: Estados Unidos, Gran Bretaña, Francia y Alemania. La duda que surge es si esos resultados son generalizables para el contexto cultural, económico y social de los consumidores españoles (Gázquez y Sánchez, 2009; Gentry et al., 2003; Martínez et al., 2010; Rosa, 2004).

Además, los precios promocionales pueden provocar modificaciones en los precios de referencia de los consumidores (Campo y Yagüe, 2007; Han et al., 2001; Krishna et al., 1991) y, en consecuencia, influir sobre sus decisiones de compra futuras (Lowe y Alpert, 2007).

El planteamiento realizado cobra gran relevancia por su repercusión directa sobre las decisiones de compra de los consumidores y, en consecuencia, sobre los objetivos y la rentabilidad de las empresas. De ahí el interés de profundizar en el estudio de las reacciones de los consumidores ante los precios promocionales.

\section{FUNDAMENTOS TEÓRICOS E HIPÓTESIS}

El objetivo fundamental de esta investigación ha consistido en identificar rasgos que definen a los consumidores que se sienten más atraídos por las ofertas basadas en precios 
(precios promocionales). En concreto, se ha analizado la influencia de la importancia del precio en sus decisiones de compra, así como de ciertas características demográficas (sexo, nivel de ingresos y nivel de formación). Así mismo, se ha considerado de interés determinar si la tendencia a adquirir productos con precios promocionales genera un mejor conocimiento de los precios por parte de los consumidores.

Son numerosos los estudios que demuestran que los consumidores que más importancia conceden a los precios son los que les prestan más atención, los que con más frecuencia utilizan este tipo de información en sus decisiones de compra, y los que muestran mayor tendencia a realizar comparaciones entre precios (Dickson y Sawyer, 1990; Gázquez y Sánchez, 2009; Krishna, 1991; Palazón y Delgado, 2008; Vanhuele y Drèze, 2002). Este tipo de comportamiento, de búsqueda y análisis de informaciones, coloca a estos consumidores sensibles a los precios en una buena situación para detectar y "aprovechar" las promociones basadas en precios que ofrecen las empresas (Krishna et al. 1991; Mulhern et al., 1998; Papatla y Krishnamurthi, 1996; Zentes et al., 2007). Se trata, pues, de un planteamiento que se corresponde con una perspectiva de análisis de coste-beneficio (DelVeccio, 2005; Martínez y Montaner, 2006): tal y como se sostiene en la teoría del nivel de adaptación, la evaluación de un precio supone la emisión de un juicio sobre el "valor otorgado al dinero" (Homburg et al., 2005). Esto implica que los consumidores poseen ciertos esquemas de referencia internos a través de los cuales responden al gasto (Niedrich et al., 2001; Ofir, 2004; Shirai, 2003).

A las motivaciones de carácter económico que mueven a los consumidores a buscar ofertas en el mercado hay que añadir otras de índole social y cultural, como son el entretenimiento asociado a la realización de las compras (Gázquez y Sánchez, 2009; Honea y Dahl, 2005; Kolodinsky, 1990; Swinyard, 1993; Urbany et al., 1996) y el reconocimiento social por el correcto desempeño del rol de optimización del presupuesto familiar (Carpenter y Moore, 2008; Higie et al., 1987; Rosa, 2004; Urbany et al., 1996). En cualquier caso, bajo estas otras motivaciones también subyace, como elemento de base, una cuestión económica: la búsqueda de las mejores opciones de compra según un criterio de minimización de costes monetarios (Choi et al., 2010; Ganesh et al., 2000). Esto implica que el precio desempeña un papel importante en la toma de decisiones.

Los argumentos anteriores permiten plantear una primera hipótesis dentro de nuestro estudio:

H1: Las personas que más importancia conceden a los precios dentro de sus decisiones de compra son las que con más frecuencia adquieren productos que están de oferta.

Por otra parte, los consumidores más sensibles a los precios tienden a buscar y analizar más informaciones sobre precios (Miranda y Kónya, 2007; Gijsbrechts et al., 2003), bien para utilizarlas en sus decisiones de compra actuales, bien para almacenarlas en su memoria en espera de nuevas decisiones de compra (Zentes et al., 2007). Dicho conocimiento puede ser, por tanto, el resultado de un aprendizaje intencional -realización de un esfuerzo de aprendizaje y memorización consciente- o de un aprendizaje incidental -como resultado fortuito del uso de la información- (Binkley y Bejnarowicz, 2003; Crespo y Del Barrio, 2010; Urbany et al., 1996).

En consecuencia, la búsqueda, el análisis y la comparación de informaciones sobre precios, con la finalidad de adquirir las ofertas más ventajosas, pueden conducir a un mejor 
conocimiento de los precios (Alba y Hutchinson, 2000; Campos y Yagüe, 2006; Dickson y Sawyer, 1990; Kukar-Kinney et al., 2007). De este modo, es probable que el precio, al ser utilizado como reclamo para atraer la atención del comprador, sea recordado con más facilidad (Krishna et al., 1991; Kukar-Kinney et al., 2007), en la medida en que "la presencia del descuento anima a prestar más interés en el precio, llevando a los individuos a evaluar el incentivo en relación a lo que pagan" (Palazón y Delgado, 2009).

Esto puede incluso llegar a crear un hábito en el comportamiento de compra (Lim et al., 2005; Urbany et al., 1996; Vanhuele y Drèze, 2002). Se trata de la propensión a la promoción, entendida como el aumento de la probabilidad de adquisición de una oferta comercial por el hecho de estar en promoción (Palazón y Delgado, 2009), y que está positivamente relacionada con la intención de búsqueda de información y la intensidad de su análisis (Crespo y Del Barrio, 2010; Gázquez y Sánchez, 2009). En concreto, la propensión a la promoción tiene capacidad para influir sobre el comportamiento de búsqueda, la elección de marca y establecimiento, el tiempo de compra, la cantidad comprada y la categoría de consumo (DelVeccio, 2005), lo que aumenta la intención de buscar nuevas promociones.

En definitiva, en la medida en que las respuestas de los consumidores ante los precios están influidas por el proceso seguido para adquirir información al respecto (WalserLuchesi, 1998), cabe esperar que los consumidores que adquieren productos que están en oferta procesen y almacenen con más exactitud la información relativa a los precios (Miyazaki et al., 2000). Tal y como señalan Crespo y Del Barrio (2010), si el consumidor se implica en la búsqueda de información y controla dicho proceso, es de esperar que procese mucho más la información que el comprador tradicional. Por tanto, "el hecho de comparar habitualmente los precios, aunque no se acuda a cada punto de venta para adquirir tan sólo los productos de oferta, contribuye a un mayor conocimiento de los precios del mercado", debido al esfuerzo realizado para buscar y comparar esas informaciones sobre precios (Urbany et al., 1996, p. 101).

La argumentación desarrollada nos lleva a plantear una segunda hipótesis dentro de nuestro estudio:

H2: Los precios de los productos que están "de oferta" se conocen con más exactitud que los precios de los productos que no lo están.

Otra cuestión interesante es conocer el perfil sociocultural y demográfico de los consumidores que tienen más tendencia a adquirir productos que están en oferta (Estelami y Lehmann, 2001; Kwon y Kwon, 2007; Krishna et al., 1991). En el presente estudio se ha recogido información sobre las siguientes características: sexo, nivel de formación y nivel de ingresos.

La inclusión del sexo se justifica por los diferentes roles que desempeñan hombres y mujeres dentro de la unidad familiar. En concreto, a pesar de la transformación de los roles de compra tradicionales que se está produciendo en España (Casares, 2003), son diversos los estudios realizados en el contexto español que revelan que siguen siendo las mujeres las que se ocupan mayoritariamente de planificar y realizar el aprovisionamiento del hogar (Castaño, 2002; Instituto Nacional de Estadística, 2003; Salido, 2002; Secretaría General de Asuntos Sociales de España, 2002). Por lo tanto, es probable que sean las mujeres las que muestren mayor tendencia a adquirir productos que están "en oferta", dada su responsabilidad como "administradoras del presupuesto familiar". 
Por lo que respecta al nivel de ingresos y al nivel de formación, existen estudios que revelan que son los consumidores de menor nivel de ingresos y formación los que centran más su atención en los precios (Estelami y Lehmann, 2001; Urbany et al., 1996; Wakefield e Inman, 1993). En el caso de los ingresos, la justificación de base es la necesidad de optimizar la utilización del presupuesto disponible. En cuanto al nivel de formación, las situaciones laborales y retributivas más favorables suelen corresponder a las personas con mayor nivel de formación. En cualquier caso, lo que resulta interesante en este estudio es comprobar si las tendencias identificadas en los contextos socio-culturales analizados en estudios previos son extensibles al caso español. En definitiva, la hipótesis planteada con respecto a las variables socio-demográficas es la siguiente:

H3: La mayor tendencia a adquirir productos que están en oferta corresponde a las mujeres, a las personas con ingresos más bajos, y también a las personas con niveles de formación bajos.

Esta hipótesis puede subdividirse del modo siguiente:

H3a: La mayor tendencia a adquirir productos que están en oferta corresponde a las mujeres, pues asumen con frecuencia la optimización del presupuesto familiar.

H3b: La mayor tendencia a adquirir productos que están en oferta corresponde a las personas con ingresos más bajos, pues disponen de presupuestos ajustados.

H3c: La mayor tendencia a adquirir productos que están en oferta corresponde a las personas con niveles de formación bajos, pues disponen de menos ingresos.

\section{VARIABLES Y MEDIDAS}

Las variables que participan en este estudio han sido medidas como se detalla a continuación.

En el caso de la importancia del precio en la decisión de compra, se ha utilizado una escala de cinco niveles, que oscilan entre los valores "nada importante" (1) y "lo más importante" (5).

Por lo que respecta al carácter promocional del precio, se ha definido una variable dicotómica con los siguientes niveles: "producto en oferta" y "producto sin oferta". Las cuantías de las ofertas de precios han oscilado entre el $10 \%$ y el $15 \%$.

En cuanto a las alternativas para evaluar el conocimiento de los precios, éstas son diversas (Estelami, 2003). Los objetivos de la investigación y el contexto en el que se ha desarrollado la recogida de los datos (dicho contexto se analiza en las secciones siguientes), ha llevado a seleccionar las siguientes:

La primera de ellas está referida al conocimiento de los precios en términos absolutos, es decir, al conocimiento de la cifra exacta (Dickson y Sawyer, 1990; Miyazaki et al., 2000). En nuestro estudio, esta medida del conocimiento de los precios equivale a un comportamiento del consumidor que consiste en fijar su atención directamente en las señales promocionales -precio reducido-(Chiang et al., 2001; Parsons, 2003).

Para evaluar la exactitud de los precios recordados por los consumidores (precios de referencia internos) se ha seleccionado la siguiente fórmula (Zeithaml, 1982), que es la más 
utilizada, y la que cuenta con mayor grado de aceptación en este ámbito de estudio (Estelami y Lehmann, 2001; Manning et al., 2003):

$$
\% \text { Error }=\mid \begin{array}{|l|}
\text { Precio recortado }- \text { Precio correcto } \\
{ } \mid }
\end{array}
$$

Esta formulación permite conocer en qué grado son correctos los precios de referencia internos utilizados por los consumidores. Dichos precios de referencia internos constituyen estándares con los que los consumidores comparan los precios observados (Gruca et al., 2002), que configuran en función del entorno de la fijación de precios (Kopalle et al., 1996; Lowengart, 2002), y que se basan en la premisa de que los consumidores responden a los precios, no de forma absoluta, sino en relación con ese elemento de referencia (Kumar et al., 1998; Mazumdar et al., 2005). Dentro de nuestro contexto de investigación, los precios de referencia vienen representados por los precios recordados por los consumidores.

La segunda medida del conocimiento de los precios utilizada en este estudio analiza dicho conocimiento en términos relativos, medido a través de la capacidad de los consumidores para ordenar distintas marcas de un producto en función de su precio (Gruca et al., 2002). Por tanto, esta medida relativa permite evaluar el grado de corrección de las comparaciones realizadas por los consumidores entre el precio promocional y otros precios (precios de referencia): el precio anterior para ese mismo producto, o bien el precio de otros productos competidores (Chen et al., 1998; Niedrich et al., 2001). En concreto, cada sujeto encuestado debía ordenar tres marcas del producto considerado. A este respecto, es preciso realizar algunas observaciones: a) una de las marcas era la que el sujeto había adquirido; b) las dos marcas restantes eran propuestas por el encuestador 3 ; c) todas las marcas se comercializaban en el punto de venta en el que se realizaba la encuesta; d) entre las tres marcas que cada encuestado debía ordenar, había una con precio promocional; e) al proponerle al entrevistado las marcas que debía ordenar, se especificaba la forma de presentación, tamaño del envase, etc., para de este modo homogeneizar al máximo el esquema de comparación.

La exactitud de la ordenación ha sido evaluada a través de tres niveles: "tres marcas correctamente ordenadas"; "una marca correctamente ordenada" y "ninguna marca correctamente ordenada" - esta metodología coincide con la utilizada en investigaciones previas: Binkley and Bejnarowicz (2003), Zeithaml (1982)-.

Por lo que respecta a las variables socio-demográficas, la única variable que requiere una explicación en cuanto a su medida es el nivel de ingresos. En concreto, dada la naturaleza de los productos analizados en el estudio empírico (productos de compra frecuente y consumo habitual en el hogar), la variable "nivel de ingresos" toma como referencia el conjunto de la unidad familiar,. No obstante, durante la realización del sondeo piloto previo a la recogida de datos definitiva, se observó una clara dificultad de los encuestados para estimar el nivel de ingresos mensual de la unidad familiar. Además, también se observó una actitud negativa y, en muchos casos, se produjo una ausencia de respuesta. Por este mo-

\footnotetext{
${ }^{3}$ Las marcas seleccionadas para cada categoría de producto se encontraban entre las más vendidas en cada tipo de establecimiento (información suministrada por los gerentes de los establecimientos). Cabe señalar que la marca con precio promocional no tenía que ser, necesariamente, la de menor precio.
} 
tivo, se decidió estimar el nivel de ingresos de cada sujeto encuestado a partir del nivel de ingresos total de la unidad familiar y del número de integrantes de la misma -la estimación del nivel de ingresos de la unidad familiar se realizó a partir de la profesión de cada uno de sus miembros- (Estelami and Lehmann, 2001; Rosa, 2004).

\section{PROCESO DE SELECCIÓN DE LA MUESTRA}

Los sujetos encuestados fueron captados dentro de los puntos de venta, o bien a la salida de los mismos, una vez que habían realizado en ellos su compra. Además, para llevar a cabo las entrevistas se consultaron los tiques de compra, lo que corroboró su condición de "compradores". Por lo que se refiere al tamaño de la muestra seleccionada (se ha seguido un procedimiento de muestreo aleatorio simple), se han obtenido un total de 600 encuestas válidas (este tamaño muestral se encuentra entre los más elevados de las investigaciones que se han realizado dentro de este ámbito de estudio). Las 600 encuestas se distribuyeron proporcionalmente entre tres tipos de establecimientos: pequeños (tiendas detallistas), medianos (supermercados) y grandes (hipermercados). Seguidamente se presenta la ficha técnica del estudio (tabla 1):

Tabla 1

\section{Ficha técnica}

\begin{tabular}{ll}
\hline \multicolumn{1}{c}{ Lugar y fechas recogida datos } & \multicolumn{1}{c}{ Sevilla. Abril-Mayo 2009 } \\
\hline Población objetivo* & Compradores de las categorías de productos analizadas \\
Varianza poblacional & $16 \%$ \\
Tamaño muestral & 600 encuestas válidas \\
Error de muestreo & $3,2 \%$ \\
Nivel de confianza & $95 \%$ \\
Método recogida datos & Encuesta personal \\
\hline
\end{tabular}

* La población objetivo ha sido considerada infinita en términos estadísticos, dado su elevado tamaño previsto (compradores de productos de consumo habitual), aunque no se disponen de datos exactos.

\section{DISEÑO DE LA RECOGIDA DE DATOS}

Seguidamente se realizarán algunas precisiones sobre las preguntas formuladas a los encuestados.

Producto: Selección de un producto dentro de la compra que el sujeto acababa de realizar. Los productos considerados en el estudio son los siguientes: café molido, café instantáneo, cacao en polvo, refrescos de cola, refrescos de naranja-limón, aceite de oliva, aceite de girasol, detergente para lavadora, detergente para lavavajillas, suavizante para la ropa, champú, gel de baño, pasta dentífrica, manzanas, kiwis, peras, tomates, leche, margarina, 
agua, yogur, zumo, atún en lata, cereales para desayuno y papel higiénico. Además, se ha constatado que para situar la duración de la entrevista en un nivel "razonable" -entre 3 y 4 minutos (Camacho et al., 2000) - debía preguntarse a cada encuestado exclusivamente por un producto.

Precio pagado: ¿Cuál es el precio que ha pagado por este producto? Si no responde, dejar en blanco -la investigación de Estelami y Lehmann (2001) demuestra que en los estudios en los que se introduce la posibilidad de que el sujeto no mencione ningún precio se reduce el error cometido al recordar los precios-. Si responde, debe dar una cifra concreta.

Precio real: Identificado a partir del tique de compra.

Importancia del precio: Medida a través de una escala de 5 puntos.

Ordenación de marcas en función de su precio: Ordene las siguientes marcas en función de su precio (de mayor a menor precio). Si no responde, dejar en blanco.

Finalizadas las cuestiones relativas al comportamiento de compra, se plantearon las que aludían a las características socio-culturales y demográficas.

\section{METODOLOGÍA UTILIZADA PARA ANALIZAR LOS DATOS}

Las técnicas utilizadas para realizar el tratamiento de los datos recogidos están relacionadas con el análisis de asociaciones mediante tablas de contingencia, así como con el análisis de la varianza (nivel de significación $=5 \%$ ).

En este último caso se ha comprobado el cumplimiento de los requisitos de independencia de las observaciones -garantizada por la forma de obtener los datos-, y homogeneidad de las varianzas - mediante la aplicación del test de Levene-. No obstante, para la variable "porcentaje de error cometido al nombrar el precio del producto", ha sido preciso realizar transformaciones para lograr el cumplimiento del supuesto de normalidad.

En concreto, se han identificado los niveles de la diferencia entre el precio nombrado y el precio real que generan los elevados valores de los coeficientes de asimetría y curtosis obtenidos (4,4 y 29,8 respectivamente). El principal valor causante de la distorsión es el 0, que indica que el precio nombrado coincide exactamente con el real. Por ello, el primer paso para lograr la normalidad de la variable dependiente ha sido eliminar los valores nulos. De este modo, se ha analizado la influencia de las variables independientes sobre las "diferencias no nulas entre el precio nombrado y el precio real".

Además, se ha efectuado una transformación consistente en calcular el logaritmo neperiano de la variable dependiente definida (Hair et al., 1999). Como resultado de estas operaciones se ha obtenido, para la nueva variable dependiente definida, respectivamente, un coeficiente de asimetría de $(-0,109)$, un coeficiente de curtosis de $(-0,0557)$ y un pvalor para la prueba de Kolmogorov-Smirnov de 0,098. Por tanto, puede afirmarse que la variable "diferencia no nula entre el precio nombrado y el precio real" cumple el supuesto de normalidad.

En la sección de resultados se indicará cuál es el p-valor obtenido si se toma como variable dependiente el logaritmo neperiano de las diferencias (no nulas) entre el precio nombrado y el precio real ( $\mathrm{p}$-valor ${ }_{1}$ ), y también cuál es el p-valor obtenido si se toma como variable dependiente esa misma variable, pero sin aplicar el logaritmo ( $\mathrm{p}$-valor ${ }_{2}$ ) -este es el procedimiento estadístico seguido por Estelami y Lehmann, 2001-. 


\section{RESULTADOS OBTENIDOS A PARTIR DE LA MUESTRA}

La primera hipótesis planteada hace referencia a la importancia del precio dentro de las decisiones de compra de los consumidores. Los resultados descriptivos obtenidos se recogen en las tablas 2 y 3 .

Tabla 2

Frecuencias y porcentajes para "Importancia del precio"

\begin{tabular}{lccc}
\hline \multicolumn{1}{c}{ Importancia del precio } & Frecuencia & Porcentaje válido & Porcentaje acumulado \\
\hline Nada importante & 21 & 3,5 & 3,5 \\
Poco importante & 134 & 22,3 & 25,8 \\
Importancia media & 181 & 30,2 & 56,0 \\
Bastante importante & 211 & 35,2 & 91,2 \\
Lo más importante & 53 & 8,8 & 100,0 \\
Total & 600 & 100,0 & \\
\hline
\end{tabular}

Tabla 3

Estadísticos descriptivos para "Importancia del precio"

\begin{tabular}{lc}
\hline N & 600 \\
Media & 3,24 \\
Mediana & 3,00 \\
Moda & 4 \\
Desv. típica & 1,01 \\
Varianza & 1,01 \\
Mínimo & 1 \\
Máximo & 5 \\
\hline
\end{tabular}

Estos resultados muestran que, dentro del ámbito del estudio, el precio adquiere una gran relevancia: el 74\% de los sujetos de la muestra opina que el precio tiene una "importancia media" $(30,2 \%)$, que es "bastante importante" $(35,2 \%)$ o que es "lo más importante" $(8,8 \%)$, frente al $26 \%$ que lo considera "poco importante" $(22,3 \%)$ o "nada importante" $(3,5 \%)$.

El objetivo directo de la investigación realizada ha consistido en comprobar si existe relación significativa entre la importancia del precio y la adquisición de productos que se encuentran en oferta. Para ello se han utilizado tablas de contingencia y medidas de asociación (Chi-cuadrado de Pearson y Razón de Verosimilitud). Los resultados obtenidos se recogen en las tablas 4 y 5 . 
Tabla 4

Tabla de contingencia Importancia precio * Producto en oferta

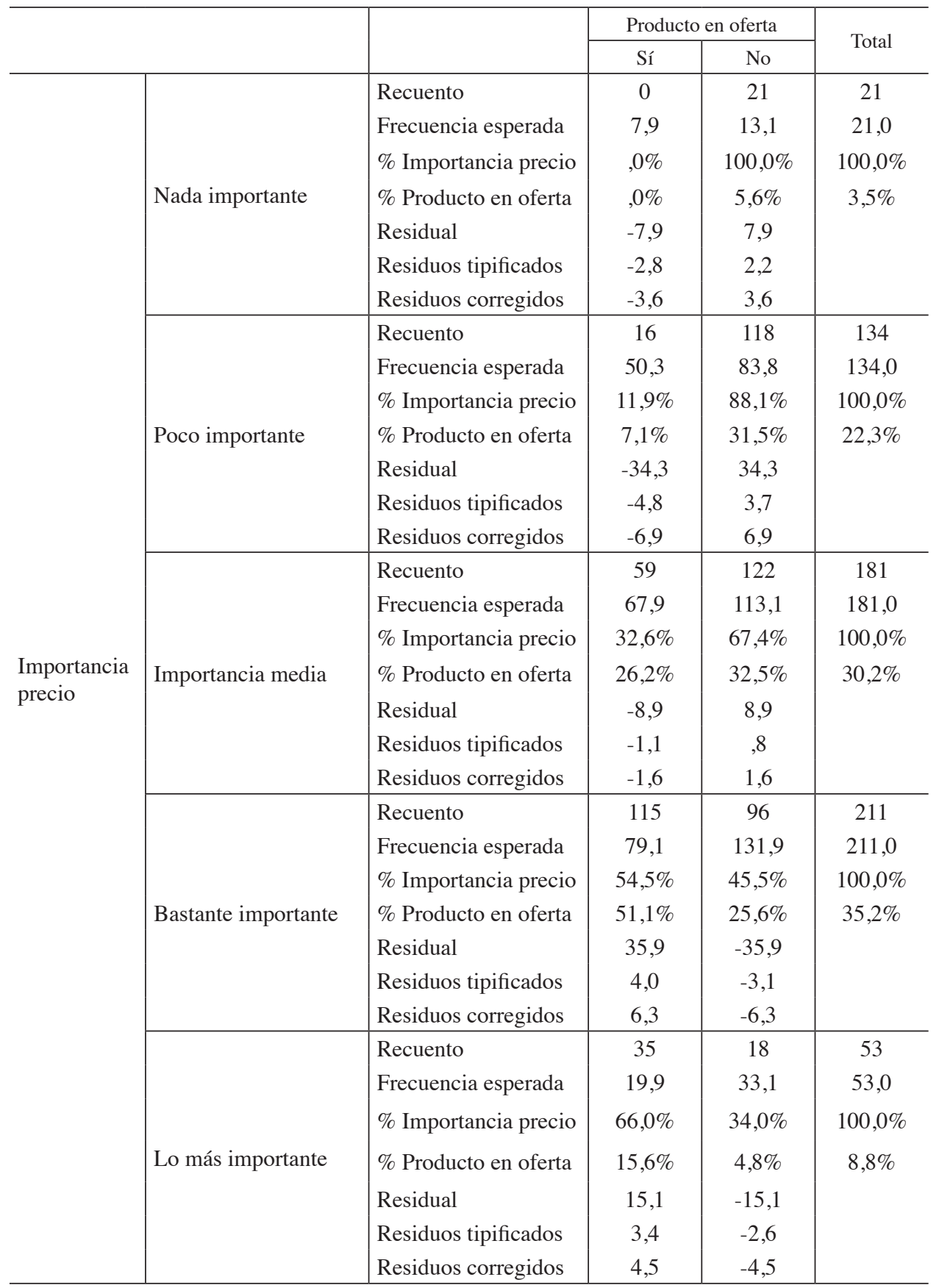




\begin{tabular}{c|l|c|c|c}
\hline \multirow{2}{*}{ Total } & & \multicolumn{2}{|c|}{ Producto en oferta } & \multirow{2}{*}{ Total } \\
\cline { 3 - 4 } & & Sí & No & \\
\hline \multirow{3}{*}{ Tecuento } & 225 & 375 & 600 \\
& Frecuencia esperada & 225,0 & 375,0 & 600,0 \\
& \% Importancia precio & $37,5 \%$ & $62,5 \%$ & $100,0 \%$ \\
& \% Producto en oferta & $100,0 \%$ & $100,0 \%$ & $100,0 \%$ \\
\hline
\end{tabular}

Tabla 5

Pruebas de chi-cuadrado

\begin{tabular}{lccc}
\hline & Valor & gl & $\begin{array}{c}\text { Sig. asint. } \\
\text { (bilateral) }\end{array}$ \\
\hline Chi-cuadrado de Pearson & 96,249 & 4 &, 000 \\
Razón de verosimilitud & 108,616 & 4 &, 000 \\
Asociación lineal por lineal & 94,495 & 1 &, 000 \\
N de casos válidos & 600 & & \\
\hline
\end{tabular}

Estos resultados indican que el porcentaje de productos en oferta adquiridos por los consumidores aumenta a medida que lo hace la importancia del precio en sus decisiones de compra. En concreto, un 37,5\% de los sujetos encuestados han adquirido productos en oferta; de ellos, el 66,7\% manifestó que el precio era "bastante importante" o "lo más importante", mientras que el 26,2\% lo consideró un factor de "importancia media". Esta tendencia se ve corroborada por los valores que alcanzan las medidas de asociación aplicadas. El p-valor $(0,000)$ indica, en todos los casos, que existe una relación estadísticamente significativa entre ambas variables.

En definitiva, se confirma la primera hipótesis planteada en el estudio: son las personas que más importancia conceden a los precios las que muestran mayor tendencia a adquirir productos con precios promocionales.

Una cuestión interesante consiste en conocer si existen categorías de producto que presenten mayor tendencia a ser adquiridas con precio promocional que otras. Los resultados obtenidos confirman esta posibilidad $\left(\chi^{2}=50,57\right.$; $\left.\mathrm{p} \leq 0.001\right)$. En concreto, el café, el cacao en polvo, las manzanas, los kiwis, la margarina o el agua son adquiridos predominantemente sin precio promocional (a pesar de existir marcas que se encontraban en oferta en el momento de la recogida de datos); en otros productos no se identifica una tendencia clara, aunque predomine el precio no promocional, y en algunos casos (refrescos de cola, aceite de oliva, atún en lata, detergente para lavavajillas y suavizante para la ropa) el número de compras con precio promocional supera al de compras sin promoción. Los resultados concretos se recogen en las tablas 6 y 7 . 
Determinantes y consecuencias de la efectividad de las promociones basadas en precios

Tabla 6

Tabla de contingencia Producto*Producto en oferta

\begin{tabular}{|c|c|c|c|c|}
\hline \multirow{2}{*}{ Producto } & \multirow{2}{*}{ Estadísticos } & & & \multirow{2}{*}{ Tota } \\
\hline & & Sí & No & \\
\hline \multirow{3}{*}{ Café } & Frecuencia observada & 2 & 13 & 15 \\
\hline & Frecuencia esperada & 5,6 & 9,4 & 15 \\
\hline & Residuos corregidos & $-2,0$ & 2,0 & \\
\hline \multirow{3}{*}{ Café soluble } & Frecuencia observada & 6 & 11 & 17 \\
\hline & Frecuencia esperada & 6,4 & 10,6 & 17 \\
\hline & Residuos corregidos & $-0,2$ & 0,2 & \\
\hline \multirow{3}{*}{ Cacao en polvo } & Frecuencia observada & 2 & 10 & 12 \\
\hline & Frecuencia esperada & 4,5 & 7,5 & 12 \\
\hline & Residuos corregidos & $-1,5$ & 1,5 & \\
\hline \multirow{3}{*}{ Refrescos cola } & Frecuencia observada & 16 & 12 & 28 \\
\hline & Frecuencia esperada & 10,5 & 17,5 & 28 \\
\hline & Residuos corregidos & 2,2 & $-2,2$ & \\
\hline \multirow{3}{*}{ Refrescos naranja/limón } & Frecuencia observada & 9 & 13 & 22 \\
\hline & Frecuencia esperada & 8,3 & 13,8 & 22 \\
\hline & Residuos corregidos & 0,3 & $-0,3$ & \\
\hline \multirow{3}{*}{ Aceite oliva } & Frecuencia observada & 17 & 15 & 32 \\
\hline & Frecuencia esperada & 12 & 20 & 32 \\
\hline & Residuos corregidos & 1,9 & $-1,9$ & \\
\hline \multirow{3}{*}{ Aceite girasol } & Frecuencia observada & 7 & 13 & 20 \\
\hline & Frecuencia esperada & 7,5 & 12,5 & 20 \\
\hline & Residuos corregidos & $-0,2$ & 0,2 & \\
\hline \multirow{3}{*}{ Detergente lavadora } & Frecuencia observada & 12 & 17 & 29 \\
\hline & Frecuencia esperada & 10,9 & 18,1 & 29 \\
\hline & Residuos corregidos & 0,4 & $-0,4$ & \\
\hline \multirow{3}{*}{ Detergente lavavajillas } & Frecuencia observada & 11 & 6 & 17 \\
\hline & Frecuencia esperada & 6,4 & 10,6 & 17 \\
\hline & Residuos corregidos & 2,4 & $-2,4$ & \\
\hline \multirow{3}{*}{ Suavizante ropa } & Frecuencia observada & 16 & 14 & 30 \\
\hline & Frecuencia esperada & 11,3 & 18,8 & 30 \\
\hline & Residuos corregidos & 1,8 & $-1,8$ & \\
\hline \multirow{3}{*}{ Champú } & Frecuencia observada & 11 & 23 & 34 \\
\hline & Frecuencia esperada & 12,8 & 21,3 & 34 \\
\hline & Residuos corregidos & $-0,6$ & 0,6 & \\
\hline
\end{tabular}




\begin{tabular}{|c|c|c|c|c|}
\hline \multirow{2}{*}{ Producto } & \multirow{2}{*}{ Estadísticos } & & & \multirow{2}{*}{ Tota } \\
\hline & & Sí & No & \\
\hline \multirow{3}{*}{ Gel de baño } & Frecuencia observada & 13 & 26 & 39 \\
\hline & Frecuencia esperada & 14,6 & 24,4 & 39 \\
\hline & Residuos corregidos & $-0,6$ & 0,6 & \\
\hline \multirow{3}{*}{ Pasta dentífrica } & Frecuencia observada & 9 & 16 & 25 \\
\hline & Frecuencia esperada & 9,4 & 15,6 & 25 \\
\hline & Residuos corregidos & $-0,2$ & 0,2 & \\
\hline \multirow{3}{*}{ Manzanas } & Frecuencia observada & 0 & 8 & 8 \\
\hline & Frecuencia esperada & 3 & 5 & 8 \\
\hline & Residuos corregidos & $-2,2$ & 2,2 & \\
\hline \multirow{3}{*}{ Kiwis } & Frecuencia observada & 0 & 12 & 12 \\
\hline & Frecuencia esperada & 4,5 & 7,5 & 12 \\
\hline & Residuos corregidos & $-2,7$ & 2,7 & \\
\hline \multirow{3}{*}{ Peras } & Frecuencia observada & 6 & 5 & 11 \\
\hline & Frecuencia esperada & 4,1 & 6,9 & 11 \\
\hline & Residuos corregidos & 1,2 & $-1,2$ & \\
\hline \multirow{3}{*}{ Leche } & Frecuencia observada & 17 & 39 & 56 \\
\hline & Frecuencia esperada & 21 & 35 & 56 \\
\hline & Residuos corregidos & $-1,2$ & 1,2 & \\
\hline \multirow{3}{*}{ Margarina } & Frecuencia observada & 6 & 22 & 28 \\
\hline & Frecuencia esperada & 10,5 & 17,5 & 28 \\
\hline & Residuos corregidos & $-1,8$ & 1,8 & \\
\hline \multirow{3}{*}{ Agua } & Frecuencia observada & 4 & 16 & 20 \\
\hline & Frecuencia esperada & 7,5 & 12,5 & 20 \\
\hline & Residuos corregidos & $-1,8$ & 1,8 & \\
\hline \multirow{3}{*}{ Yogur } & Frecuencia observada & 19 & 20 & 39 \\
\hline & Frecuencia esperada & 14,6 & 24,4 & 39 \\
\hline & Residuos corregidos & 1,5 & $-1,5$ & \\
\hline \multirow{3}{*}{ Zumo } & Frecuencia observada & 15 & 22 & 37 \\
\hline & Frecuencia esperada & 13,9 & 23,1 & 37 \\
\hline & Residuos corregidos & 0,4 & $-0,4$ & \\
\hline \multirow{3}{*}{ Atún en lata } & Frecuencia observada & 12 & 11 & 23 \\
\hline & Frecuencia esperada & 8,6 & 14,4 & 23 \\
\hline & Residuos corregidos & 1,5 & $-1,5$ & \\
\hline \multirow{3}{*}{ Cereales desayuno } & Frecuencia observada & 6 & 18 & 24 \\
\hline & Frecuencia esperada & 9 & 15 & 24 \\
\hline & Residuos corregidos & $-1,3$ & 1,3 & \\
\hline
\end{tabular}




\begin{tabular}{l|l|c|c|c}
\hline \multirow{2}{*}{ Producto } & \multicolumn{1}{|c|}{ Estadísticos } & \multicolumn{2}{|c}{} & \multirow{2}{*}{ Total } \\
\cline { 3 - 4 } & & Sí & No & \\
\hline \multirow{3}{*}{ Papel higiénico } & Frecuencia observada & 7 & 8 & 15 \\
& Frecuencia esperada & 5,6 & 9,4 & 15 \\
& Residuos corregidos & 0,7 & $-0,7$ & \\
\hline \multirow{2}{*}{ Tomates } & Frecuencia observada & 2 & 5 & 7 \\
& Frecuencia esperada & 2,6 & 4,4 & 7 \\
\hline \multirow{2}{*}{ Total } & Residuos corregidos & 0,7 & $-0,7$ & \\
& Frecuencia observada & 225 & 375 & 600 \\
\hline
\end{tabular}

Tabla 7

Pruebas de chi-cuadrado

\begin{tabular}{lccc}
\hline & Valor & gl & $\begin{array}{c}\text { Sig. asint. } \\
\text { (bilateral) }\end{array}$ \\
\hline Chi-cuadrado de Pearson & 50,572 & 24 &, 001 \\
Razón de verosimilitud & 58,112 & 24 &, 000 \\
Asociación lineal por lineal &, 673 & 1 &, 412 \\
N de casos válidos & 600 & & \\
\hline
\end{tabular}

Al considerar los productos agrupados en "alimenticios" y "no alimenticios", la relación anteriormente mencionada desaparece, es decir, no existe asociación entre la categoría de producto y el carácter promocional del precio $(\mathrm{p}>0,05)$.

Una última cuestión destacable es la ausencia de diferencias significativas en la tendencia de los encuestados a adquirir productos con precios promocionales según los tipos de establecimientos en los que se han recogido los datos.

La segunda hipótesis planteada hace referencia al nivel de conocimiento de los precios por parte de los consumidores. En concreto, el interés se centra en la posible relación entre el carácter promocional del precio y la exactitud con la que se conoce ese precio.

Como ya se indicó previamente, para evaluar el conocimiento de los precios se han utilizado dos medidas. La primera de ellas refleja el porcentaje de error cometido por cada sujeto cuando nombra el precio del producto por el que se le ha preguntado, y que acaba de adquirir.

A este respecto, cabe destacar que el 78,3\% de los encuestados nombró un precio cuando se le solicitó. Además, el $20 \%$ del total de encuestados nombraron el precio correcto. Este porcentaje se eleva hasta el $25,5 \%$ si se calcula con respecto al número de precios nombrados.

Para evaluar la posible relación entre el porcentaje de error (no nulo) cometido al nombrar el precio y el carácter promocional del precio, se ha aplicado un test t-Student. Los resultados se recogen en las tablas 8 y 9 . 
Tabla 8

Estadísticos del grupo

\begin{tabular}{l|c|c|c|c|c}
\hline & Producto en oferta & N & Media & Desv. típ. & $\begin{array}{c}\text { Error típ. de la } \\
\text { media }\end{array}$ \\
\hline valor absoluto & Sí & 114 &, 0525 & $4,918 \mathrm{E}-02$ & $4,607 \mathrm{E}-03$ \\
error cometido & No & 233 &, 1005 & $8,969 \mathrm{E}-02$ & $5,876 \mathrm{E}-03$ \\
\hline \multirow{2}{*}{ LNVABE1B } & Sí & 114 & $-3,2998$ &, 8471 & $7,934 \mathrm{E}-02$ \\
& No & 233 & $-2,6685$ &, 9063 & $5,937 \mathrm{E}-02$ \\
\hline
\end{tabular}

Tabla 9

Prueba de muestras independientes

\begin{tabular}{|c|c|c|c|c|c|c|c|c|c|}
\hline & \multicolumn{2}{|c|}{$\begin{array}{c}\text { Prueba } \\
\text { Levene } \\
\text { igualdad } \\
\text { varianzas }\end{array}$} & \multicolumn{7}{|c|}{ Prueba T para la igualdad de medias } \\
\hline & $\mathrm{F}$ & Sig. & $\mathrm{t}$ & $\mathrm{gl}$ & $\begin{array}{c}\text { Sig. } \\
\text { (bilateral) }\end{array}$ & $\begin{array}{c}\text { Dif. } \\
\text { medias }\end{array}$ & $\begin{array}{c}\text { Error típ } \\
\text { diferencia }\end{array}$ & $\begin{array}{r}\text { Interval } \\
\text { para } 1\end{array}$ & $\begin{array}{l}\text { o de confianza } \\
\text { a diferencia }\end{array}$ \\
\hline & & & & & & & & Inferior & Superior \\
\hline $\begin{array}{l}\text { valor absoluto } \\
\text { error cometido }\end{array}$ & 24,54 & ,000 & $-5,32$ & 345 & ,000 &,- 0479 & $9,001 \mathrm{E}-03$ &,- 0656 & $-3,0202 \mathrm{E}-02$ \\
\hline LNVABE1B & 668 & ,414 & $-6,225$ & 345 &, 000 &,- 6313 & ,1014 &,- 8308 &,- 4319 \\
\hline
\end{tabular}

Los p-valores obtenidos $\left(\mathrm{t}_{1}=-5,322 ; \mathrm{p}\right.$-valor ${ }_{1}=0,000 ; \mathrm{t}_{2}=-6,225 ; \mathrm{p}$-valor $\left.{ }_{2}=0,000\right)$ indican que existe una influencia significativa del carácter promocional de los precios sobre el porcentaje de error cometido al recordarlos. Más concretamente, los precios de los productos que están de oferta se estiman con más exactitud que los precios de los productos que no lo están: $5,3 \%$ de error frente a $10,1 \%$ respectivamente.

Además, cuando los encuestados nombraron el precio correcto (porcentaje de error nulo), en el 68,2\% de los casos el producto estaba en oferta, y en el 31,8\% no lo estaba. Estos resultados confirman la segunda hipótesis planteada en el estudio, cuando se evalúa el conocimiento de los precios a través del recuerdo de la cifra exacta -precios absolutos-.

El conocimiento de los precios también ha sido evaluado en este estudio en términos relativos (ordenación de tres marcas en función de su precio). Un primer resultado interesante muestra que el $61,8 \%$ de los encuestados nombró una ordenación. Si se compara este resultado con el correspondiente a la primera medida del conocimiento de los precios (el $78,3 \%$ de los encuestados nombró un precio), existe una diferencia porcentual importante.

En cuanto a la relación entre el carácter promocional del precio y la exactitud de las ordenaciones de marcas, los resultados obtenidos indican que los mayores porcentajes de ordenaciones absolutamente correctas y los menores porcentajes de ordenaciones absolutamente incorrectas corresponden a los productos que están en oferta $(50,8 \%$ y 9,9\% respectivamente para los productos que están de oferta, frente a $42,2 \%$ y $16,1 \%$ para los que 
no lo están). No obstante, los valores de las medidas de asociación indican que la relación entre las variables analizadas no es estadísticamente significativa ( $\mathrm{p}>0,05)$. Por tanto, si bien para la primera medida del conocimiento de los precios aplicada (recuerdo del precio exacto) se concluye la existencia de una influencia significativa del carácter promocional del precio sobre el conocimiento del mismo, no ocurre lo mismo para la segunda medida del conocimiento de los precios utilizada (ordenación de marcas en función de su precio).

Para concluir el análisis del conocimiento de los precios, cabe mencionar que no se han observado diferencias significativas según el tipo de establecimiento considerado.

Una última cuestión analizada en este estudio se centra en la posible relación entre ciertas características socio-demográficas de los consumidores (sexo, nivel de ingresos y nivel de formación) y su tendencia a adquirir productos que estén en oferta.

En primer lugar, los estadísticos descriptivos reflejan que la muestra seleccionada está integrada, de forma predominante, por mujeres y por personas con ingresos medios; en el caso del nivel de formación, la distribución es bastante homogénea. En cuanto a las relaciones planteadas, seguidamente se detallan los resultados obtenidos:

a) Entre las variables sexo y producto en oferta existe una relación estadísticamente significativa, tal y como reflejan los datos recogidos en las tablas 10 y 11:

Tabla 10

Tabla de contingencia Producto en oferta * Sexo

\begin{tabular}{|c|c|c|c|c|c|}
\hline & & & & & Totol \\
\hline & & & Hombre & Mujer & Iotal \\
\hline & & Recuento & 60 & 165 & 225 \\
\hline & & Frecuencia esperada & 78,4 & 146,6 & 225,0 \\
\hline & & $\%$ de Producto oferta & $26,7 \%$ & $73,3 \%$ & $100,0 \%$ \\
\hline & Sí & $\%$ de Sexo & $28,7 \%$ & $42,2 \%$ & $37,5 \%$ \\
\hline & & Residual & $-18,4$ & 18,4 & \\
\hline & & Residuos tipificados & $-2,1$ & 1,5 & \\
\hline Producto en & & Residuos corregidos & $-3,3$ & 3,3 & \\
\hline oferta & & Recuento & 149 & 226 & 375 \\
\hline & & Frecuencia esperada & 130,6 & 244,4 & 375,0 \\
\hline & & $\%$ Producto en oferta & $39,7 \%$ & $60,3 \%$ & $100,0 \%$ \\
\hline & No & $\%$ de Sexo & $71,3 \%$ & $57,8 \%$ & $62,5 \%$ \\
\hline & & Residual & 18,4 & $-18,4$ & \\
\hline & & Residuos tipificados & 1,6 & $-1,2$ & \\
\hline & & Residuos corregidos & 3,3 & $-3,3$ & \\
\hline & & Recuento & 209 & 391 & 600 \\
\hline$T$ & & Frecuencia esperada & 209,0 & 391,0 & 600,0 \\
\hline lotal & & $\%$ de Producto oferta & $34,8 \%$ & $65,2 \%$ & $100,0 \%$ \\
\hline & & $\%$ de Sexo & $100,0 \%$ & $100,0 \%$ & $100,0 \%$ \\
\hline
\end{tabular}


Tabla 11

Pruebas de chi-cuadrado

\begin{tabular}{lccccc}
\hline & Valor & gl & $\begin{array}{c}\text { Sig. asint. } \\
\text { (bilateral) }\end{array}$ & $\begin{array}{c}\text { Sig. exacta } \\
\text { (bilateral) }\end{array}$ & $\begin{array}{r}\text { Sig. exacta } \\
\text { (unilateral) }\end{array}$ \\
\hline $\begin{array}{l}\text { Chi-cuadrado de Pearson } \\
\begin{array}{l}\text { Corrección de } \\
\text { continuidad(a) }\end{array}\end{array}$ & 10,577 & 1 &, 001 & & \\
$\begin{array}{l}\text { Razón de verosimilitud } \\
\text { Estadístico exacto de Fisher }\end{array}$ & 10,009 & 1 &, 002 & & \\
$\begin{array}{l}\text { Asociación lineal por lineal } \\
\text { N de casos válidos }\end{array}$ & 10,792 & 1 &, 001 & &, 001 \\
\hline
\end{tabular}

Como puede observarse, el 28,7\% de los hombres encuestados compran productos que están en oferta, frente al 42,2\% de las mujeres. Por tanto, las mujeres muestran mayor tendencia que los hombres a adquirir productos con precios promocionales.

b) Entre las variables nivel de ingresos y producto en oferta existe una relación estadísticamente significativa. Los datos se presentan en las tablas 12 y 13.

Tabla 12

Tabla de contingencia Producto en oferta * nivel ingresos

\begin{tabular}{|c|c|c|c|c|c|c|}
\hline & & & & vel ingre & & \\
\hline & & & Bajo & Medio & Alto & Iotal \\
\hline & & Recuento & 80 & 96 & 49 & 225 \\
\hline & & Frecuencia esperada & 60,4 & 97,5 & 67,1 & 225,0 \\
\hline & & $\%$ de Producto oferta & $35,6 \%$ & $42,7 \%$ & $21,8 \%$ & $100,0 \%$ \\
\hline & Sí & $\%$ de nivel ingresos & $49,7 \%$ & $36,9 \%$ & $27,4 \%$ & $37,5 \%$ \\
\hline & & Residual & 19,6 & $-1,5$ & $-18,1$ & \\
\hline & & Residuos tipificados & 2,5 &,- 2 & $-2,2$ & \\
\hline Producto & & Residuos corregidos & 3,7 &,- 3 & $-3,3$ & \\
\hline en oferta & & Recuento & 81 & 164 & 130 & 375 \\
\hline & & Frecuencia esperada & 100,6 & 162,5 & 111,9 & 375,0 \\
\hline & & $\%$ de Producto oferta & $21,6 \%$ & $43,7 \%$ & $34,7 \%$ & $100,0 \%$ \\
\hline & No & $\%$ de nivel ingresos & $50,3 \%$ & $63,1 \%$ & $72,6 \%$ & $62,5 \%$ \\
\hline & & Residual & $-19,6$ & 1,5 & 18,1 & \\
\hline & & Residuos tipificados & $-2,0$ & , 1 & 1,7 & \\
\hline & & Residuos corregidos & $-3,7$ &, 3 & 3,3 & \\
\hline
\end{tabular}




\begin{tabular}{l|l|c|c|c|c}
\hline \multirow{2}{*}{} & & \multicolumn{3}{|c|}{ Nivel ingresos } & \multirow{2}{*}{ Total } \\
\cline { 3 - 5 } & & Bajo & Medio & Alto & \\
\hline \multirow{3}{*}{ Total } & Recuento & 161 & 260 & 179 & 600 \\
& Frecuencia esperada & 161,0 & 260,0 & 179,0 & 600,0 \\
& \% de Producto oferta & $26,8 \%$ & $43,3 \%$ & $29,8 \%$ & $100,0 \%$ \\
& $\%$ de nivel ingresos & $100,0 \%$ & $100,0 \%$ & $100,0 \%$ & $100,0 \%$ \\
\hline
\end{tabular}

Tabla 13

Pruebas de chi-cuadrado

\begin{tabular}{l|c|c|c}
\hline & Valor & gl & Sig. asint. (bilateral) \\
\hline Chi-cuadrado de Pearson & $18,074(\mathrm{a})$ & 2 &, 000 \\
Razón de verosimilitud & 18,119 & 2 &, 000 \\
Asociación lineal por lineal & 17,882 & 1 &, 000 \\
N de casos válidos & 600 & & \\
\hline
\end{tabular}

Los datos muestran que el 49,7\% de los encuestados con un nivel de ingresos bajo compra productos que están "de oferta", frente al 36,9\% de los que tienen ingresos medios, y al $27,4 \%$ de los que poseen un nivel de ingresos alto. Por tanto, son las personas con menor nivel de ingresos las que se muestran más proclives a adquirir las ofertas.

c) Entre las variables nivel de formación y producto en oferta existe una relación estadísticamente significativa (tablas 14 y 15).

Tabla 14

Tabla de contingencia Producto en oferta * Nivel formación

\begin{tabular}{|c|c|c|c|c|c|c|c|}
\hline & & & \multicolumn{4}{|c|}{ Nivel formación } & \multirow{2}{*}{ Total } \\
\hline & & & Sin estudios & Primarios & Secudarios & Superiores & \\
\hline \multirow{7}{*}{$\begin{array}{l}\text { Producto } \\
\text { en oferta }\end{array}$} & \multirow{7}{*}{ Sí } & Recuento & 53 & 77 & 54 & 41 & 225 \\
\hline & & Frecuencia esperada & 48,8 & 61,5 & 58,5 & 56,3 & 225,0 \\
\hline & & $\%$ de Producto oferta & $23,6 \%$ & $34,2 \%$ & $24,0 \%$ & $18,2 \%$ & $100,0 \%$ \\
\hline & & $\%$ de Nivel formación & $40,8 \%$ & $47,0 \%$ & $34,6 \%$ & $27,3 \%$ & $37,5 \%$ \\
\hline & & Residual & 4,3 & 15,5 & $-4,5$ & $-15,3$ & \\
\hline & & Residuos tipificados & ,6 & 2,0 &,- 6 & $-2,0$ & \\
\hline & & Residuos corregidos & ,9 & 2,9 &,- 9 & $-3,0$ & \\
\hline
\end{tabular}




\begin{tabular}{|c|c|c|c|c|c|c|c|}
\hline & & & \multicolumn{4}{|c|}{ Nivel formación } & \multirow{2}{*}{ Total } \\
\hline & & & Sin estudios & Primarios & Secudarios & Superiores & \\
\hline \multirow{7}{*}{$\begin{array}{l}\text { Producto } \\
\text { en oferta }\end{array}$} & \multirow{7}{*}{ No } & Recuento & 77 & 87 & 102 & 109 & 375 \\
\hline & & Frecuencia esperada & 81,3 & 102,5 & 97,5 & 93,8 & 375,0 \\
\hline & & $\%$ de Producto oferta & $20,5 \%$ & $23,2 \%$ & $27,2 \%$ & $29,1 \%$ & $100,0 \%$ \\
\hline & & $\%$ de Nivel formación & $59,2 \%$ & $53,0 \%$ & $65,4 \%$ & $72,7 \%$ & $62,5 \%$ \\
\hline & & Residual & $-4,3$ & $-15,5$ & 4,5 & 15,3 & \\
\hline & & Residuos tipificados &,- 5 & $-1,5$ &, 5 & 1,6 & \\
\hline & & Residuos corregidos &,- 9 & $-2,9$ & ,9 & 3,0 & \\
\hline \multirow{4}{*}{\multicolumn{2}{|c|}{ Total }} & Recuento & 130 & 164 & 156 & 150 & 600 \\
\hline & & Frecuencia esperada & 130,0 & 164,0 & 156,0 & 150,0 & 600,0 \\
\hline & & $\%$ de Producto oferta & $21,7 \%$ & $27,3 \%$ & $26,0 \%$ & $25,0 \%$ & $100,0 \%$ \\
\hline & & $\%$ de Nivel formación & $100,0 \%$ & $100,0 \%$ & $100,0 \%$ & $100,0 \%$ & $100,0 \%$ \\
\hline
\end{tabular}

Tabla 15

Pruebas de chi-cuadrado

\begin{tabular}{l|c|c|c}
\hline & Valor & gl & Sig. asint. (bilateral) \\
\hline Chi-cuadrado de Pearson & $14,012(\mathrm{a})$ & 3 &, 003 \\
Razón de verosimilitud & 14,158 & 3 &, 003 \\
Asociación lineal por lineal & 9,257 & 1 &, 002 \\
N de casos válidos & 600 & & \\
\hline
\end{tabular}

En concreto, las personas con mayor nivel de formación adquieren menos productos que están "en oferta" que las personas con un nivel de formación menor (el 40,8\% de los encuestados sin estudios y el $47 \%$ de los encuestados con estudios primarios compran productos que están "de oferta", frente al 34,6\% de los sujetos con estudios secundarios y al $27,3 \%$ de los que poseen estudios superiores).

Estos resultados confirman la hipótesis 3. Efectivamente, son las mujeres y las personas con niveles bajos de ingresos y de formación las que con más frecuencia adquieren productos con precios promocionales.

\section{CONCLUSIONES E IMPLICACIONES PARA LA GESTIÓN EMPRESARIAL}

A partir del estudio realizado es posible extraer un conjunto de conclusiones de utilidad para la gestión empresarial en general, y para la gestión de precios y promociones en particular. 
En primer lugar, para las categorías de productos analizadas, todas ellas de compra y consumo habitual, el precio ha sido considerado un factor importante en las decisiones de compra de muchos consumidores. Por lo tanto, también debe ser un factor de decisión prioritario para las empresas que comercializan este tipo de bienes.

Además, la confirmación de la primera hipótesis del estudio indica que cuanto mayor es la importancia atribuida al precio por los consumidores, mayor es su tendencia a adquirir marcas que estén en oferta. Por lo tanto, la importancia del precio parece corresponderse con el rol negativo del mismo - los precios elevados disminuyen la posibilidad de compra(Lichtenstein et al., 1993).

No obstante, el grado de importancia del precio y la tendencia a adquirir productos en oferta ha sido desigual según la categoría de producto considerada. Estas diferencias deben generar estrategias de precios adaptadas: cuando el precio desempeña un papel importante en las decisiones de los compradores, las actuaciones de las empresas en materia de precios serán mucho más visibles, y tendrán un impacto mayor sobre los clientes y sobre la imagen de precio de los puntos de venta, que no parece estar vinculada al tamaño de los mismos. Del mismo modo, si el precio no se perfila como un factor determinante para los consumidores en la categoría de producto considerada, las estrategias de marketing deberán centrarse en otras variables, como pueden ser la fidelidad a la marca o la calidad del servicio.

Una vez constatado que los compradores más sensibles al precio son los que más centran su atención en las ofertas, surge un nuevo interrogante: ¿qué aspectos concretos de la oferta son los que atraen esa atención?

Un resultado interesante en este sentido está vinculado a la forma de presentar la promoción (Martínez et al., 2010). En concreto, en nuestro estudio, la mayoría de encuestados dispone de precios de referencia internos, lo que puede explicarse por la frecuencia de compra y uso de los artículos analizados. Además, esas referencias internas consisten con más frecuencia en valores absolutos (cifras exactas) que relativos (ordenaciones entre precios). Ahora bien, esos niveles de referencia también son, en muchas ocasiones, incorrectos, siendo las referencias internas absolutas más exactas que las relativas, es decir, más próximas a sus valores reales.

Este resultado es de gran relevancia, pues los precios recordados condicionan las evaluaciones de los precios, tanto si son exactos como si no lo son. Por tanto, resulta recomendable que las empresas presenten sus promociones con valores numéricos concretos que indiquen la magnitud del beneficio promocional (Choi et al., 2010; Palazón y Delgado, 2009).

Más exactamente, cuando se adquiere un producto que está "en oferta", la información relativa a la cifra del precio, que actúa como reclamo, impacta de forma directa sobre los consumidores. Este anuncio parece ser suficiente para muchos de ellos al tomar sus decisiones de compra, por lo que no consideran necesario establecer comparaciones entre el precio promocional y el precio de otras alternativas de compra. De ahí la importancia de diseñar adecuadamente las estrategias de promociones basadas en precios, y de utilizar en ese diseño informaciones sobre el grado de conocimiento que tienen los consumidores acerca de los precios de los productos que comercializa la empresa (Greenleaf, 1995; Inman y McAlister, 1993; Kahn y Raju, 1991; Kalwani y Yim, 1992; Kim et al., 1999; Krishna et al. 1991; Mulhern y Padgett, 1995).

La argumentación anterior enlaza directamente con los precios de referencia externos o de contexto (Biswas y Blair, 1991; Cooper, 1969; Mondéjar et al., 2010; Puto, 1987; Ra- 
jendran y Tellis, 1994; Rosa, 2002; Yadav y Seiders, 1998). Se trata de las informaciones promocionales anunciadas por las empresas, cuya finalidad es proporcionar al consumidor un punto de referencia que aumente su valor percibido con relación al producto (Cebollada y Múgica, 1997; Oubiña, 1997), y que aumente también su credibilidad (Blair y Landon, 1981; Burman y Biswas, 2004; Danny y Lee, 2005; Urbany et al., 1988). Esa credibilidad sólo será factible cuando los precios de referencia externos suministrados por las empresas no entren en conflicto con las referencias internas de los consumidores, que en nuestro estudio son, mayoritariamente, incorrectas. En definitiva, la gestión correcta de las promociones de precios exige a las empresas conocer qué referencias utilizan sus clientes en sus evaluaciones, y cómo establecen las comparaciones entre los precios de referencia y los precios actuales de sus productos, ya que esa comparación constituye un indicador de la respuesta del mercado ante un ajuste de precios.

Por tanto, las promociones de precios no representan exclusivamente herramientas tácticas utilizadas para estimular las ventas de un producto en un cierto período de tiempo, sino que también constituyen herramientas estratégicas, en la medida en que inciden sobre los procesos de evaluación y percepción de los precios por parte de los consumidores, y pueden llegar a modificar sus estándares de referencia en esas evaluaciones (Campo y Yagüe, 2007).

Otra tendencia interesante detectada indica que las mujeres compran más productos en promoción que los hombres. Una posible explicación para este hecho puede ser que las mujeres continúan asumiendo más responsabilidad que los hombres en las tareas relacionadas con el hogar y con la optimización del presupuesto familiar, a pesar de que las diferencias de roles cada vez son menores (Ekström, 2003; Martín, 2003; McGinnis et al., 2003; McGoldrick et al., 1999; Putrevu, 2001; Secretaría General de Asuntos Sociales, 2002; Shankarmahesh et al., 2003). Este argumento es confirmado por el predominio de mujeres dentro de la muestra utilizada, a pesar de la aleatoriedad aplicada en la selección de los sujetos. Finalmente, los sujetos con menores niveles de formación suelen corresponderles niveles de ingresos más bajos, lo que parece hacerles más sensibles a los precios y a las ofertas que les realizan las empresas. En estos casos, la vinculación del precio a sacrificio económico potencia la efectividad de las promociones.

Dada la importancia estratégica de estos planteamientos, subrayamos la importancia para las empresas de contar con informaciones que les ayuden a optimizar el diseño y la efectividad de sus acciones promocionales, y a valorar las consecuencias reales de dichas promociones (Kamins et al., 2009; Shankar y Krishnamurthi, 1996). De ahí la relevancia de estudios como el presentado.

\section{LIMITACIONES Y LÍNEAS FUTURAS DE INVESTIGACIÓN}

Las investigaciones referidas a la determinación y gestión de los precios desde el punto de vista de la empresa (identificación de políticas de precios óptimas a lo largo del tiempo, efecto de las curvas de experiencia, venta conjunta y separada de bienes, etc.) proporcionan una base útil en el establecimiento de reglas normativas para la fijación de los precios. No obstante, existe un importante déficit de investigaciones que conduzcan al desarrollo de modelos que incorporen los aspectos psicológicos y de comportamiento del consumidor 
ante los precios (Tsiros y Hardesty, 2010). En este sentido, el estudio realizado aporta información relevante acerca del comportamiento de los consumidores ante los precios promocionales. No obstante, dada la amplitud de este campo de investigación, surgen nuevas cuestiones que resulta interesante responder.

En primer lugar, se ha observado que existe mayor tendencia por parte de los compradores a adquirir en oferta ciertas categorías de productos. La pregunta que surge es ésta: ¿por qué? El interrogante planteado nos conduce hacia la conveniencia de introducir en este ámbito de estudio otras variables, como son la fidelidad a la marca, la lealtad al punto de venta, y las asociaciones entre precio y calidad (Gázquez y Sánchez, 2009).

Otra cuestión interesante consiste en diversificar las categorías de productos incluidas en el análisis (productos duraderos, servicios, ...), así como realizar análisis específicos para categorías de productos consideradas de forma individual y estudios de los posibles efectos cruzados de las promociones de precios sobre las ventas de otras categorías de productos (Parreño et al., 2009; Caplliure et al., 2010).

Por otra parte, las empresas no deben olvidar que los precios promocionales pueden influir sobre los precios de referencia internos de los compradores, y también sobre sus intervalos de precios aceptables. De ahí la necesidad de conocer cómo se produce esa influencia, y qué factores se ven implicados.

Finalmente, las diferencias culturales pueden generar comportamientos heterogéneos en los compradores (Griffin et al., 2000; Estelami et al., 2001; McGowan \& Sternquist, 1998; Mooij and Hofstede, 2002), lo que sugiere la necesidad de investigaciones futuras que evalúen el comportamiento del consumidor ante los precios promocionales en diferentes contextos geográficos y culturales.

\section{BIBLIOGRAFÍA}

ALBA, J.W. y HUTCHINSON, J.W. (2000), "Knowledge calibration: what consumers know and what they think they know", Journal of Consumer Research, Vol. 27 №. 2, pp. 123-156.

BINKLEY, J.K. y BEJNAROWICZ, J. (2003): "Consumer price awareness in food shopping: the case of quantity surcharges". Journal of Retailing, 79(1), pp. 27-35.

BISWAS, A. y BLAIR, E.A. (1991): "Contextual effects of reference prices in retail advertisements". Journal of Marketing, Vol. 55, julio, pp. 1-12.

BLAIR, E.A. y LANDON, E.L. JR. (1981): «The effects of reference prices in retail advertisements», Journal of Marketing, Vol. 45, primavera, pp. 61-69.

BURMAN, B. y BISWAS, A. (2004): "Reference prices in retail advertisements: moderating effects of market price dispersion and need for cognition on consumer value perception and shopping intention". The Journal of Product and Brand Management 13 (6), pp. 379-389.

CAMACHO, C.; PRADO, C.; ROMERO, B. y VALERA, S. (2000): “¿Cómo hacen encuestas los encuestadores?". Metodología de Encuestas, Vol. 2, №. 1, pp. 117-133.

CAMPO, S. y YAGÜE, M.J. (2007): "Effects of price promotions on the perceived price". Journal of Service Management, 18 (3), pp. 269-286.

CAPLLIURE, E.; MIQUEL, M.J., y PÉREZ, C. (2010): "La elección de la marca del distribuidor en productos duraderos: factores de influencia". Cuadernos de Gestión, Vol. 10, pp. 125-147. CARPENTER, J. y MOORE, M. (2008): “US consumers' perceptions of non-price retail promotions". International Journal of Retail and Distribution Management, 36 (2), pp. 111-123.

CASARES, J. (2003): “El nuevo paisaje del consumo”. Distribución y Consumo, 70, pp. 5-10. 
CASTAÑO COLLADO, C. (2002): "Los cambios en el hogar y el trabajo doméstico". Segundo Seminario Internacional sobre Género y Urbanismo Infraestructuras para la Vida Cotidiana (ETSAM, UPM). Madrid.

CEBOLLADA PASCUAL, J.; MÚGICA GRIJALBA, J.M. (1997): "La gestión de los precios y promociones en la empresa minorista". Distribución y Consumo, abril-mayo, pp. 77-87.

CHEN, S.S.; MONROE, K.B. y LOU, Y. (1998): "The effects of framing price promotion messages on consumers' perceptions and purchase intentions". Journal of Retailing, 74, 3, pp. 353-372.

CHIANG, J.; CHUNG, C. y CREMERS, E.T. (2001): "Promotions and the pattern of grocery shopping time". Journal of Applied Statistics, Vol.28, Nº. pp. 801-819.

CHOI, S.; GE, X. y MESSINGER, P.R. (2010): "Consumer perceptions of ambiguous price promotions: scratch and save promotions versus tensile price claims". The Journal of Product and Brand Management, 19(7), pp. 477-486.

COOPER, P. (1969): "Subjective economics: factors in a psychology of spending", en Taylor, B. y Wills, G. (eds.) (1969): Princing strategy. Staples, London, pp. 112-121.

CRESPO ALMENDROS, E. y DEL BARRIO GARCÍA, S. (2010): "Influencia de la propensión a la promoción en el procesamiento de la promoción de ventas online". XXII Congreso nacional de Marketing (AEMARK). Oviedo, 22 al 24 Septiembre.

DANNY, Y.L. y LEE, M. (2005): "Consumers' Evaluations of Online Reference Price Advertisements". International Journal of Commerce \& Management, 15 (2), pp. 101-112.

DELVECCHIO, D. (2005): "Deal-prone consumers' response to promotion: the effects of relative and absolute promotion value". Psychology and Marketing, 22 (5), pp. 373-391.

DHAR, S.K. y HOCH, S.J. (1996): "Price discrimination using in-store merchandising”. Journal of Marketing, Vol. 60, enero, pp. 17-30.

DICKSON, P.R. y SAWYER, A.G. (1990): “The price knowledge and search of supermarket shoppers". Journal of Marketing, Vol. 54, julio, pp. 42-53.

EKSTRÖM, K.M. (2003): "Revisiting the family free: Historical and future consumer behavior research". Academy of Marketing Science Review, 1, (on-line journal).

ESTELAMI, H. (2003). "The effect of price presentation tactics on consumer evaluation effort of multidimensional prices". Journal of Marketing Theory and Practice, 11 (2), pp. 1-16.

ESTELAMI, H.; LEHMANN, D.R. y HOLDEN, A.C. (2001): "Macro-economic determinants of consumer price knowledge: A meta-analysis of four decades of research". International Journal of Research in Marketing, 18, pp. 341-355.

ESTELAMI, H. y LEHMANN, D.R. (2001):“The impact of research design on consumer price recall accuracy:an integrative review". Journal of Academy of Marketing Science, 29,1, pp. 36-49.

GANESH, J.; ARNOLD, M.J. y REYNOLDS, K.E. (2000): "Understanding the customer base of service providers: an examination of the differences between switchers and stayers". Journal of Marketing, Vol. 64, julio, pp. 65-87.

GÁZQUEZ ABAD, J.C. y SÁNCHEZ PÉREZ, M. (2009): "Characterising the deal-proneness of consumers by analysis of price". The International Review of Retail, Distribution and Consumer Research, 19, pp. 1-28.

GENTRY, J.W.; COMMURI, S. y JUN, S. (2003). Review of Literature on Gender in the Family. Academy of Marketing Science Review, 1, (on-line journal).

GIJSBRECHTS, E.; CAMPO, K. y GOOSSENS, T. (2003): "The impact of store flyers on store trafficand store sales: a geo-marketing approach". Journal of Retailing, 79, pp. 1-16.

GREENLEAF, E.A. (1995): "The impact of reference price effects on the profitability of price promotions". Marketing Science, Vol. 14, N. 1, invierno, pp. 82-104.

GREWAL, D.; HARDESTY, D.M. y IYER, G.R. (2004), "The effects of buyer identification and purchase timing on consumers' perceptions of trust, price fairness, and repurchase intentions", Journal of Interactive Marketing, Vol. 18 №. 4, pp. 87-101. 
GRIFFIN, M.; BABIN, B.J. y MODIANOS, D. (2000): "Shopping values of Russian consumers: the impact of habituation in a developing economy". Journal of Retailing, 76(1), pp. 33-52.

GRUCA, T.S.; SUDHARSHAN, D. y KUMAR, K.R. (2002): "Sibling brands, multiple objectives, and response to entry: The case of Marion retail coffee market". Journal of the Academy of Marketing Science, 30 (1), pp. 59-69.

HAIR, J.F.; ANDERSON, R.E.; TATHAM, R.L. y BLACK, W.C. (1999): Análisis multivariante. Ed. Prentice Hall, Madrid.

HAN, S.; GUPTA, S y LEHMANN, D.R. (2001): "Consumer price sensitivity and price thresholds", Journal of Retailing, 77 (4), pp. 435-56.

HIGIE, R.A.; FEICK, L.F. y PRICE, L.L. (1987): "Types and amount of word-of-mouth communications about retailers". Journal of Retailing, 63, pp. 260-278.

HOMBURG, C. y STOCK, R.M.(2005), "Exploring the conditions under wich salesperson work satisfaction can lead to customer satisfaction", Psychology \& Marketing, 22 (5), pp. 393-421.

HONEA, H. y DAHL, D. (2005): "The promotion affect scale: defining the affective dimensions of promotion". Journal of Business Research, 58 (3), pp. 543-551.

INMAN, J.J. y MCALISTER, L. (1993): "A retailer promotion policy model considering promotion signal sensitivity". Marketing Science, Vol. 12, Nº. 4, otoño, pp. 339-356.

KAHN, B.E. y RAJU, J.S. (1991): "Effects of price promotions on variety-seeking and reinforcement behavior". Marketing Science, Vol. 10, N. 4, otoño, pp. 316-337.

KALWANI, M.U. y YIM, H.J.R. (1992): “Consumer price and promotion expectations: an experimental study". Journal of Marketing Research, Vol. 29, febrero, pp. 90-100.

KAMINS, M.A.; FOLKES, V.S. y FEDORIKHIN, A. (2009): "Promotional Bundles and Consumers' Price Judgments: When the Best Things in Life Are Not Free", Journal of Consumer Research, vol. 36 (4), pp. 660-670.

KIM, B.; SRINIVASAN, K. y WILCOX, R.T. (1999): "Identifying price sensitive consumers: the relative merits of demographic vs. purchase pattern information". Journal of Retailing, Vol. 75, No. 2, pp. 173-193.

KLAPPER, D.; EBLING, C. y TEMME, J. (2005): "Another look at loss aversion in brand choice data: Can we characterize the loss averse consumer?". International Journal of Research in Marketing, Vol. 22, 3, pp. 239-254.

KOLODINSKI, J. (1990): "Time as a direct source of utility: The case of price information search for groceries". Journal of Consumer Affairs, 24, pp. 89-109.

KOPALLE, P.K.; RAO, A.G. y ASSUNÇAO, J.L. (1996): “Asymmetric reference price effects and dynamic pricing policies". Marketing Science, Vol. 15, No. 1, pp. 60-85.

KRIDER, R.E. y HAN, S. (2004): "Promotion Thresholds: Price Change Insensitivity or Risk Hurdle?". Canadian Journal of Administrative Sciences, Vol. 21, 3, pp. 255-272.

KRISHNA, A. (1991): "Effect of dealing patterns on consumer perceptions of deal frecuency and willingness to pay". Journal of Marketing Research, Vol. 28, noviembre, pp. 441-451.

KRISHNA, A.; CURRIM, I.S. y SHOEMAKER, R.W. (1991): "Consumer perceptions of promotional activity". Journal of Marketing, Vol. 55, abril, pp. 4-16.

KUKAR-KINNEY, M.; WALTERS, R.G. y MACKENZIE, S.B. (2007): "Consumer responses to characteristics of price-matching guarantees: The moderating role of price consciousness". Journal of Retailing, 83 (2), pp. 211-221.

KUMAR, V.; KARANDE, K. y REINARTZ, W.J. (1998): "The impact of internal and external reference prices on brand choice: the moderating role of contextual variables". Journal of Retailing, Vol. 74, No. 3, otoño, pp. 401-426.

KWON, K-N. y KWON, Y. (2007): "Demographics in sales promotion proneness: a sociocultural approach". Advances in Consumer Research, 34, pp. 288-294.

LIM, J.; CURRIM, J. y ANDREWS, R. (2005): "Consumer heterogeneity in the longer-term effects of price promotions". International Journal of Research in Marketing, 22 (4), pp. 441-457. 
LOWE, B. y ALPERT, F. (2007): "Measuring reference price perceptions for new product categories: which measure is best ?". Journal of Product and Brand Management, 16 (2), pp. 132-41.

LOWENGART, O. (2002). "Reference price conceptualisations: An integrative framework of analysis". Journal of Marketing Management, 18, pp. 145-171.

MANNING, K.C.; SPROTT, D.E. y MIYAZAKI, A.D. (2003). "Unit price usage knowledge: Conceptualization empirical assessment". Journal of Business Research, 56, pp. 367- 377.

MARTÍN CERDEÑO, V.J. (2003). "El consumidor español: factores que determinan su comportamiento". Distribución y Consumo, 70, pp. 11-29.

MARTÍNEZ, E. y MONTANER, T. (2006): "The effect of consumer's psychographic variables upon deal-proneness". Journal of Retailing and Consumer Services, 13, pp. 157-168.

MARTÍNEZ, M.P.; JIMÉNEZ, A.I., e IZQUIERDO, A. (2010): “Análisis de la satisfacción máxima del consumidor en establecimientos de alimentación: la influencia del folleto publicitario". Cuadernos de Gestión, Vol. 10, pp. 63-83. MAZUMDAR, T.; RAJ, S.P. y SINHA, I. (2005). "Reference price research: review and propositions". Journal of Marketing, 69 (October), pp. 84-102.

MCGINNIS, L., CHUN, S., y MCQUILLAN, J. (2003). "A Review of Gendered Consumption in Sport and Leisure". Academy of Marketing Science Review, 5, (on-line journal).

MCGOLDRICK, P.J., BETTS, E.J. y WILSON, A.F. (1999): "Modelling consumer price cognition: evidence from discount superstore sectors". The Service Industries Journal, 19, pp. 171-193.

MCGOWAN, K.M. y STERNQUIST, B.J. (1998): "Dimensions of price as a marketing universal: a comparison of japanese and U.S. consumers". Journal of International Marketing, 6, pp. 49-65.

MIRANDA, M. y KÓNYA, L. (2007): "Directing store flyers to the appropriate audience". Journal of Retailing and Consumer Services, 14 (3), pp. 175-181.

MIYAZAKI, A.D.; SPROTT, D.E. y MANNING, K.C. (2000): "Unit prices on retail shelf labels: an assessment of information prominence". Journal of Retailing, 76(1), pp. 93-112.

MONDÉJAR JIMÉNEZ, J.A.; MOLLÁ DESCALS, A.; GÓMEZ BORJA, M.A. y CARRICANO, M. (2010) "Formación de precios de referencia durante el proceso de compra: Un experimento simulado por ordenador". XXII Congreso nacional de Marketing (AEMARK). Oviedo, 22 al 24 Septiembre.

MOOIJ, M. y HOFSTEDE, G. (2002): "Convergence and divergence in consumer behavior: implications for international retailing". Journal of Retailing, 78(1), pp. 61-69.

MULHERN, F.J. y PADGETT, D.T. (1995): "The relationship between retail price promotions and regular price purchases". Journal of Marketing, Vol. 59, No. 4, octubre, pp. 83-92.

MULHERN, F.J.; WILLIAMS, J.D. y LEONE, R.P. (1998): "Variability of brand price elasticities across retail stores: ethnic, income, and brand determinants". Journal of Retailing, 74, 3, pp. 427-446.

NIEDRICH, R. W.; SHARMA, S. y WEDELL, D. H. (2001). "Reference Price and Price Perceptions. A Comparison of Alternative Models". Journal of Consumer Research, 28 (December), pp. 339-354.

NIEDRICH, R.W.; SHARMA, S. y WEDELL, D.H. (2001), "Reference price and price perceptions: A comparison of alternative models", Journal of Consumer Research, Vol. 28 No. 3, pp. 339-354.

OFIR, C. (2004), "Reexaminig Latitude of Price Acceptability and Price Thresholds: Predicting Basic Consumer reaction to Price", Journal of Consumer Research, Vol. 30, pp. 612-621.

OUBIÑA BARBOLLA, J. (1997): "La percepción de precios de los consumidores". Distribución y Consumo, abril-mayo, pp. 88-100.

PALAZÓN VIDAL, M. y DELGADO BALLESTER, E. (2008): "El efecto de la preocupación por el precio en la efectividad de los descuentos en precio y los regalos promocionales". XX Encuentro de Profesores Universitarios de Marketing. Maspalomas (Gran Canaria), 17-19 Septiembre.

PALAZÓN VIDAL, M. y DELGADO BALLESTER, E. (2009): "La evaluación de las promociones monetarias y no monetarias según la propensión a la promoción del consumidor". Revista Española de Investigación de marketing ESIC, Vol. 13 (1), pp. 35-70. 
PAPATLA, P. y KRISHNAMURTHI, L. (1996): “Measuring the dynamic effects of promotions on brand choice". Journal of Marketing Research, Vol. XXXIII, febrero, pp. 20-35.

PARREÑO SELVA, J; LEEFLANG, P.S.H. y VAN DOORN, J. (2009): “Efecto de las promociones del precio sobre la venta de productos no promocionados: papel moderador del tipo de visita del cliente. Revista Española de Investigación de Marketing ESIC, Vol. 13 (2) pp. 73-90.

PARSONS, A.G. (2003): "Assessing the effectiveness of shopping mall promotions: customer analysis". International Journal of Retail \& Distribution Management, Vol. 31, №. 2, pp. 74-79.

PUTO, C.P. (1987): “The Framing of Buying Decisions", Journal of Consumer Research, Vol. 14, diciembre, pp. 301-315.

PUTREVU, S. (2001): "Exploring the Origins and Information Processing Differences Between Men and Women: Implications for Advertisers". Academy of Marketing Science Review, 10, (on-line journal).

RAJENDRAN, K.N. y TELLIS, G.J. (1994): "Contextual and temporal components of reference price". Journal of Marketing, Vol. 58, No. 1, pp. 22-38.

ROSA DÍAZ, I.M. (2002). "La adopción del euro: un cambio en los sistemas de referencia del consumidor”. XII Jornadas Luso-Espanholas de Gestao Científica, 4, pp. 1-10. Portugal: Universidade da Beira Interior.

ROSA DÍAZ, I.M. (2004): "Price knowledge: Effects of Consumers' Attitudes towards Prices, Demographics, and Socio-cultural Characteristics". Journal of Product and Brand Management, Vol. 13, 6, pp. 406-428.

SALIDO, O. (2002): La participación laboral de las mujeres en España: cifras para un debate. Unidad de Políticas Comparadas (CSIC). Documento de Trabajo 02-15. Universidad Complutense de Madrid.

SARALEGUI, J. y SEOANE, P. (1999): "La familia española según el panel de hogares de la UE”. Revista Fuentes Estadísticas, 37, (on-line journal).

SECRETARÍA GENERAL DE ASUNTOS SOCIALES (2002). Las mujeres en cifras: 1996-2002. Madrid.

SHANKAR, V. y KRISHNAMURTHI, L. (1996): "Relating price sensitivity to retailer promotional variables and pricing policy: an empirical analysis". Journal of Retailing, 72, 3, pp. 249-272.

SHANKARMAHESH, M.N.; FORD, J.B. y LA TOUR, M.S. (2003): "Cultural Dimensions of Switching Behavior in Importer-Exporter Relationships". Academy of Marketing Science Review, 3, (on-line journal).

SHIRAI, M. (2003), "An analysis of multi-dimensional internal reference prices", Advances in Consumer Research, Vol. 30, pp. 258-263.

SWINYARD, W.R. (1993): "The effects of mood, involvement and quality of store experience on shopping intentions". Journal of Consumer Research, Vol. 20, septiembre, pp. 271-280.

TSIROS, M. Y HARDESTY, D.M. (2010): "Ending a Price Promotion: Retracting It in One Step or Phasing It Out Gradually", Journal of Marketing, Vol. 74 (1), pp. 49-64.

URBANY, J.E.; BEARDEN, W.O. y WEILBAKER, D.C. (1988): "The effect of plausible and exaggerated reference prices on consumer perceptions and price search". Journal of Consumer Research, Vol. 15, junio, pp. 95-110.

URBANY, J.E.; DICKSON, P.R. y KALAPURAKAL, R. (1996): "Price search in the retail grocery market”. Journal of Marketing, Vol. 60, abril, pp. 91-104.

VANHUELE, M. y DRÈZE, X. (2002). "Measuring the price knowledge shoppers bring to the store". Journal of Marketing, 66, pp. 72-85.

WAKEFIELD, K.L. y INMAN, J.J. (1993): "Who are the price vigilantes? An investigation of differentiating characteristics influencing price information processing”. Journal of Retailing, Vol. $69, \mathrm{~N}^{\circ} .2$, verano, pp. 216-233.

WALSER-LUCHESI, A. (1998): "Les phenomènes perceptuels du prix". Revue Française du Marketing, $\mathrm{N}^{\circ}$. 170, pp. 5-25. 
WELLS, J.D; PARBOTEEAH, V. y VALACICH, J.S. (2011): "Online Impulse Buying: Understanding the Interplay between Consumer Impulsiveness and Website Quality". Journal of the Association for Information Systems, 12 (1), pp. 32-56.

WINER, R.S. (1988): "Behavioral perspective on pricing: buyers' subjective perceptions of price revisited", en Devinney, T.M. (ed.) (1988a): Issues in pricing: theory and research. Lexington Books, Lexington, Massachusetts, pp. 35-57.

YADAV, M.S. y SEIDERS, K. (1998): "Is the price right? Understanding contingent processing in reference price formation". Journal of Retailing, Vol. 74, N. 3, otoño, pp. 311-329.

ZEITHAML, V.A. (1982): "Consumer response to in-store price information environments". Journal of Consumer Research, Vol. 8, marzo, pp. 357-369.

ZENTES, J.; MORSCHETT, D. y SCHRAMM-KLEIN, H. (2007): Strategic retail management: text and international cases. Wiesbaden, Germany: Gabler. 
